\title{
Spectrophotometric Determination of Aspirin and Pyridoxine Hydrochloride Using 9- Chloroacridine Reagent in Aqueous Solution
}

\author{
Intisar A. Shihab ${ }^{1}$; Theia'a N. Al-Sabha ${ }^{2 *}$ \\ ${ }^{1}$ Department of Chemistry, College of Education for Girls, University of Mosul, Mosul, Iraq \\ $2^{*}$ Department of Chemistry, College of Education for Pure Science, University of Mosul, Mosul, Iraq \\ Email: ${ }^{1}$ entesar@uomosul.edu.iq, ${ }^{2 *}$ dr_theiaa@yahoo.co.uk
}

(Received July 16, 2019; Accepted October 24, 2019; Available online March 01, 2020)

DOI: 10.33899/edusj.2019.125898.1003, (C) 2020, College of Education for Pure Science, University of Mosul.

This is an open access article under the CC BY 4.0 license (http://creativecommons.org/licenses/by/4.0/).

\begin{abstract}
:
This research paper tackles a developed method for Aspirin and Pyridoxine drugs determination in their pure forms and pharmaceutical formulations. It is based on the formation of nucleophilic substitution reaction between given drugs and 9-Chloroacridine forming a reddish orange colours. The products show maximum absorption at $532 \mathrm{~nm}$ and $534 \mathrm{~nm}$ for Aspirin and Pyridoxine respectively. The method was adhering to the Beer's law over concentration range 0.2-18 and 0.4-24 $\mu \mathrm{g} / \mathrm{ml}$ with molar absorptivity values $1.1 \times 10^{4}$ and $7 \times 10^{3} 1 . \mathrm{mol}^{-1} . \mathrm{cm}^{-1}$ and average recovery $99.87 \%$ and $100.00 \%$ for the given drugs respectively. No observed interferences appeared from the excipients commonly existed in pharmaceuticals. Successfully, application for both Aspirin and Pyridoxine was conducted in their pharmaceutical formulations. The continuous variations and the slope ratio of the products between Aspirin and pyridoxine hydrochloride were followed with 9-chloroacridine. The ratio of 1: 1 was obtained between the above two drugs and the reagent The stability constant of the products was $6.38 \times 10^{4}$ and $9.25 \times 10^{4} 1 /$ mol for both Aspirin and Pyridoxine Hydrochloride products respectively indicating the good stability of these products.
\end{abstract}

Keywords: nucleophilic substitution; Spectrophotometry; Aspirin, Pyridoxine, 9-Chloroacridin

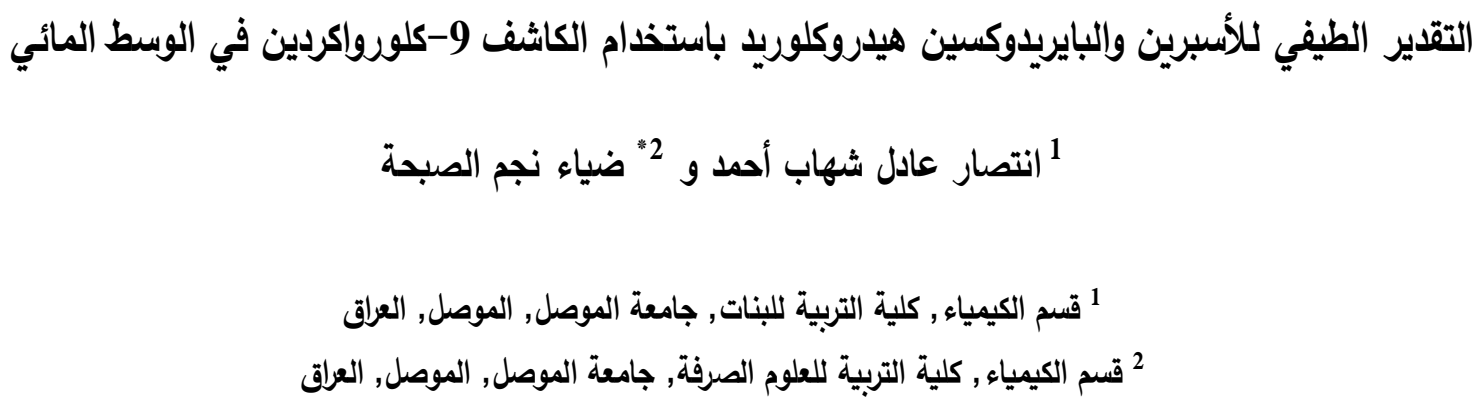

الخلاصة

تم تطوير طريقة طيفية بسـيطة في تقدير أدوية الأسبرين والبايريدوكسين بأشـكالهما النقية وفي مستحضـراتهما الصـيدلانية. اعتمدت

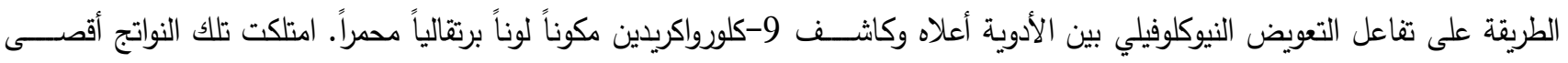
امتصاص عند الأطوال الموجية 532 و534 نانوميتر لكل من الأسبرين والبايريدوكسين على التوالي. لقد أمكن تطبيق قانون بير في مدى التراكيز

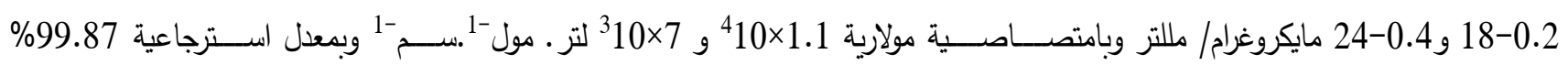

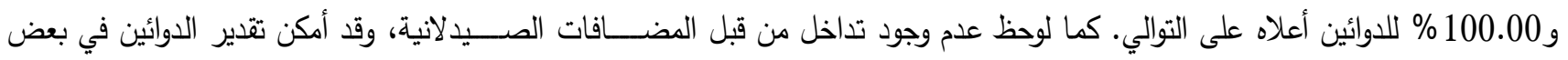


مستحضراتهما الصيدلانية بنجاح. تم إتباع طريقتي التغيرات المستمرة ونسبة الميل للناتج المتكون بين الأسبرين والبايريدوكسين هيدروكلوريد مع 9-كلورواكريدين. تم الحصول على نسبة 1:1 بين الداوئين أعلاه والكاشف وكنلك تم احتساب ثابت استترار الناتجين المتكونين بنسبة مولية 1:1.

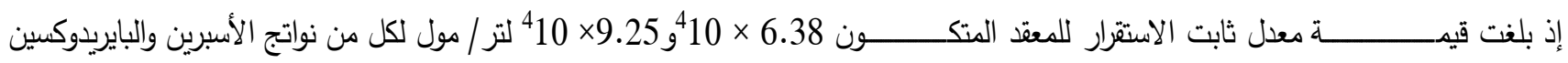
هيدروكلوريد على التوالي مما يذل على الاستقرار الجيد لتلك النواتج. الكلمات المفتاحية: التعويض النيوكلوفيلي ، مطياف فوتومتري، اسبرين، بايريدوكسين، 9- كلورواكريدين

\section{المقدمة}

البايريدوكسين هيدركلوريد: يطلق على البايريدوكسين هيدروكلوريد اسم فيتامين B وهو عبارة عن مسحوق ذي لون ابيض له ذوبانية

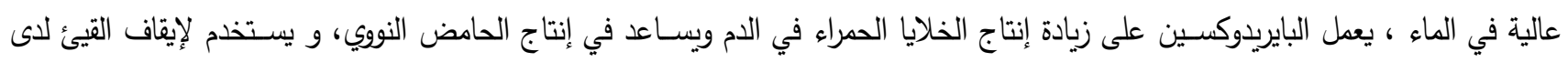

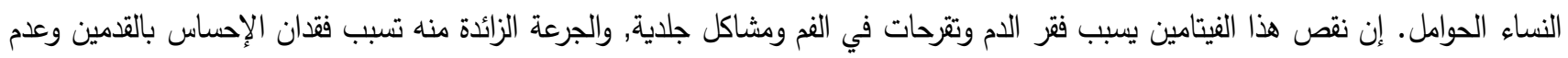
التوازن وتلف بالأعصاب (1)، يمتلك البايريدوكسين الصيغة التركيبية الأتية (2).<smiles>Cc1ncc(CO)c(CO)c1O</smiles>

Mwt $=205.638 \mathrm{~g} / \mathrm{mol}$

(5-hydroxy-6-methyl pyridine-3,4-diyl) dimethanol hydrochloride

الأسبرين: هو دواء يحتوي على المادة الفعالة اسيتايل حامض السالسيليك، يستخدم للحد من الحمى أو الالتهاب ولعلاج النوبات القلبية والسكتات الاماغية والذبحة الصدرية او منعها، يمكن تتاول الأسبرين دون مضغ أو تكسير، هناك حالات مرضية لا يسمح الطبيب للمريض تناول الأسبرين

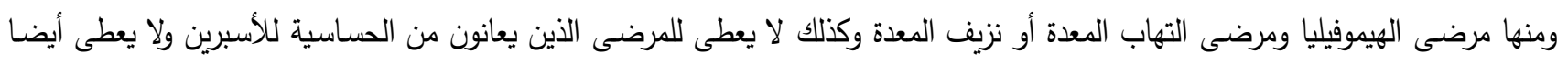

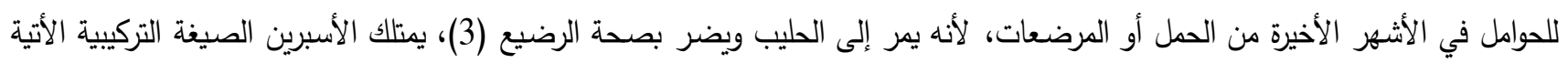

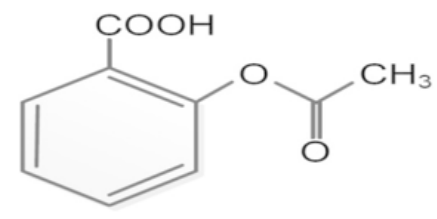

Mwt $=180.159 \mathrm{~g} / \mathrm{mol}$

Aspirin; ACETYLSALICYLIC ACID; 2-Acetoxybenzoic acid

استخدمت طرائق مختلفة لتقدير الأسبرين والبايريدوكسين هيدروكلوريد . وتضـنت هذه الطرائق طرائق طيفية (5-9) وكروماتوغرافية

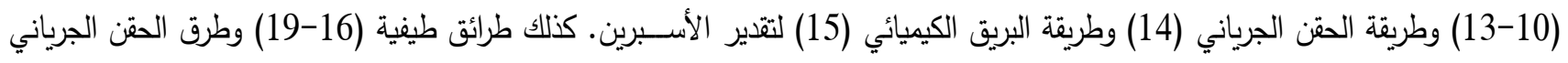
(20-21) وطريقة طيف المشــتقة (22) وطرق فولتامترية (23-24) وطرق كروماتوغرافية (25-27) لتقدير البايريدوكســين هيدروكلوريد. لتدئ تميزت تلك الطرائق باستخدام أجهزة معقدة وغالية الثمن في حين تميزت بعض الطرائق الأخرى بقلة الحساسية أو استخدام كواشف متعددة وغير 
اقتصــادية وتسـتهلك وقت في عملية التقدير ـ لذا كان الهذف من هذا البحث تطوير طريقة طيفية بسـيطة وحسـاســة لتقدير كل من الأسـبرين

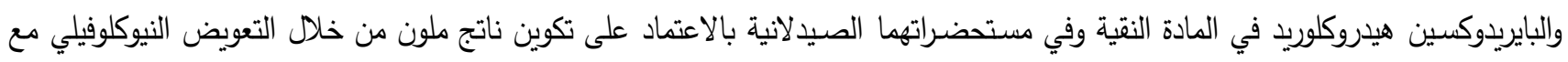
الكاشف 9-كلوروأكردين.

\section{الأجززة المستخدمة}

T92UVspectrophotometer PG instrument تمت القياسـات الطيفية بوسـاطة جهاز المطياف الفوتومتري ثنائي الحزمة نوع واستخدمت خلايا زجاجية نوع glass ذات عرض1سم، وأجريت عمليات الوزن باستخدام ميزان حساس نوع (KERN ABS) كما تمت عمليات

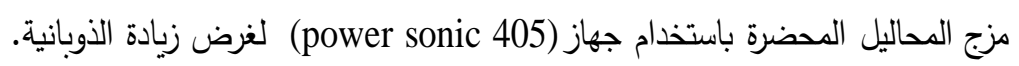
محاليل المواد الكيميائية

كانت جميع المواد الكيميائية المستخدمة على درجة عالية من النقاوة ومجهزة من شركة Fluka. محلولا الأسبرين والبايريدوكسين (100مايكروغرام/مللتر) حضرا بإذابة 0.010 غم من المادة النقية وأذيبا في كمية قليلة من الإيثانول ثم نقلا إلى قنينة حجمية سعة 100 مللتر وأكمل الحجم إلى حد العلامة بالماء المقطر . محلول 9-كلورواكريدين (4.6×10-3 مولاري) حضر بإذابة 0.0985 غم من المادة النقية في كمية قليلة من الماء المقطر ثم نقل إلى قنينة حجمية سعة 100 مللتر وأكمل الحجم إلى حد العلامة بالماء المقطر .

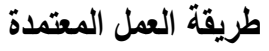

أضيف إلى مجموعة قنان حجمية سعة 25 مللتر حجوم متزايدة (ملترات) من كل من الأسبرين والبايريدوكسين هيدروكلوريد وبتركيز

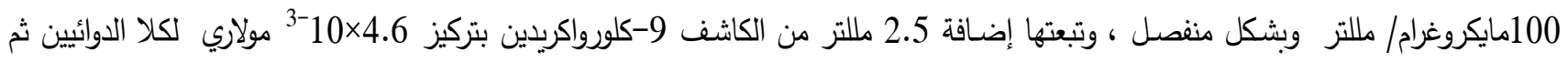

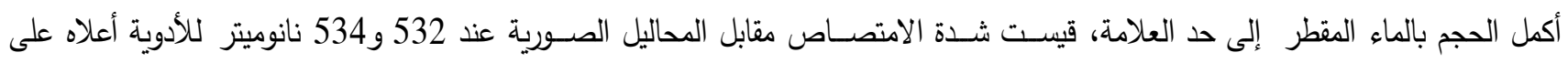
التوالي وعند درجة حرارة المختبر (28)م). - - تحليل أقراص الأسبرين 75ملفف و 81 ملفم و و 100 ملفم

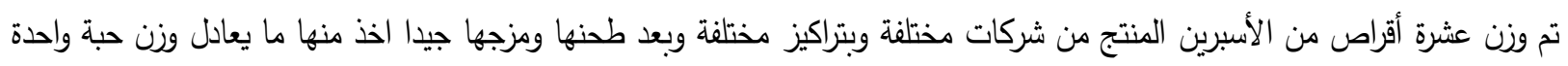

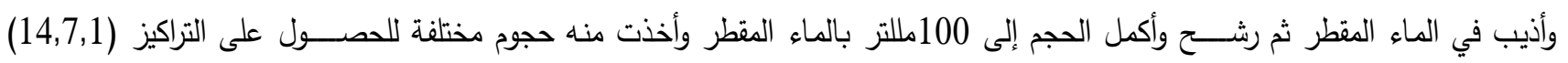
مايكروغرام/ مللتر من الأسبرين، وتم بعدها تم إيجاد تركيز المركب الدوائي باستخدام معادلة الخط المستقيم للمنحني القياسي للمادة النقية.

\section{- تحليل حقن فيتامين Bo} اخذ 0.5 ملتر من محتوى الحقنة وخفق إلى 100مللتر للحصــول على التركيز 250 مايكروغرام/مللتر ، ثم حضـر منه محلول بتركيز

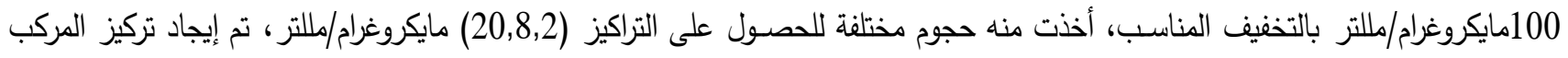
الدوائي في الحقنة باستخدام معادلة الخط المستقيم للمنحني القياسي للمادة النقية.

- تحليل أقراص البايريلوكسين هيلروكلوريد (50 ملفم)

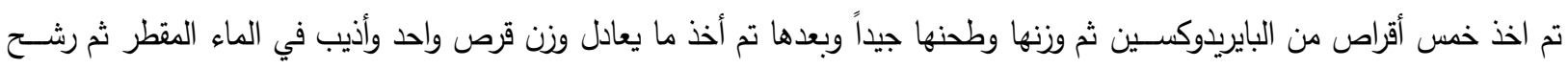

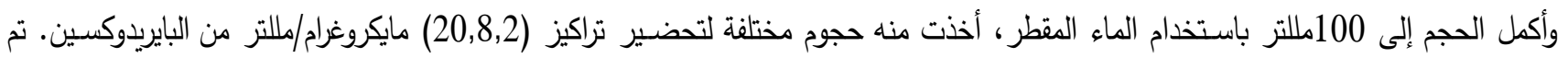
إيجاد تركيز المركب الدوائي باستخدام معادلة الخط المستقيم للمنحني القياسي للمادة النقية. 


\section{النتائج والمناقشة}

طيف الامتصاص

بهدف تطوير طريقة طيفية بسيطة وحساسـة لتقدير الأسبرين والبايريدوكسين هيدروكلوريد تم رسم طيفا الامتصساص لناتج المركبين الدوائيين مع الكاشف 9-كلوروأكردين وفي الوسط المائي عند اطوال موجية تراوحت بين 350-600 نانوميتر . لقد وجد ان أعلى امتصـاص لتلك لكائ النواتج عند 532 و534 نانوميتر لكلا الدوائين أعلاه على التوالي، في حين وجد أن المحلول الصوري يعطي اعلى امتصاص عند الطول الموجي 468نانوميتر ، الثكل (1) يوضح طيفا الامتصاص النهائية.

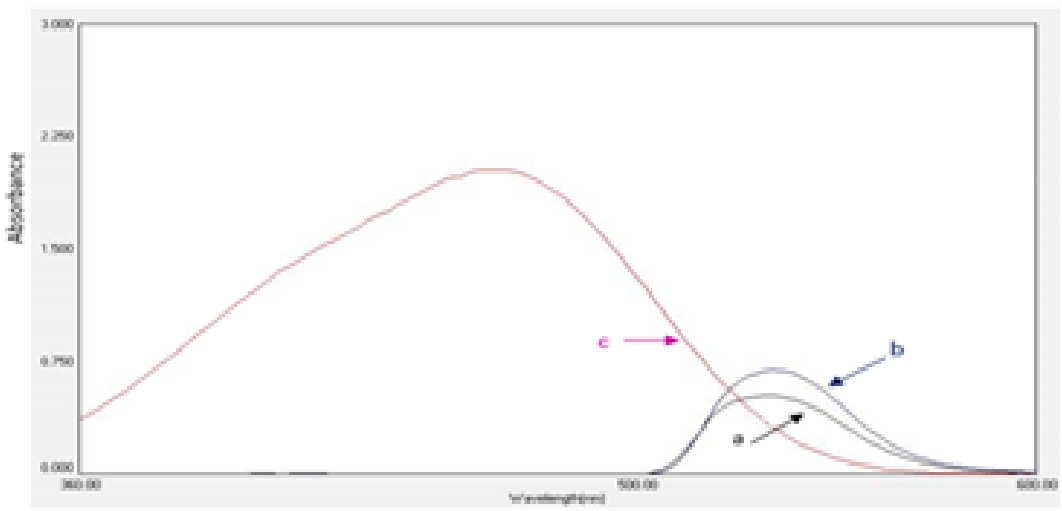

شكل (1) أطياف الامتصاص: a- ناتج تفاعل 8 مايكروغر ام/ مللتر اسبرين مع 2.5مللتر من 9- كلورو اكريدين مقابل المحلول الصوري.

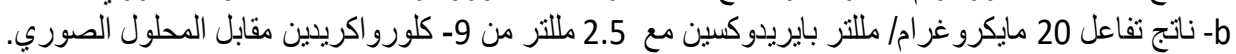

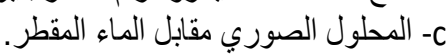

الظروف المثلى ضبط أجريت التجارب اللاحقة باســخذام 4 مايكروغرام/مللتر من الأسـبرين و8 مايكروغرام/ مللتر من البايريدوكسـين هيدروكلوريد في حجم نهائي مقداره 25 مللتر . تأثير الدالة الحامضية تمت دراسة تأثير الدالة الحامضية على تكوين الناتج لكلا الدوائين وذلك بإضافة حجوم متزايدة (0-5 مللتر ) من حامض الهيدروكلوريك

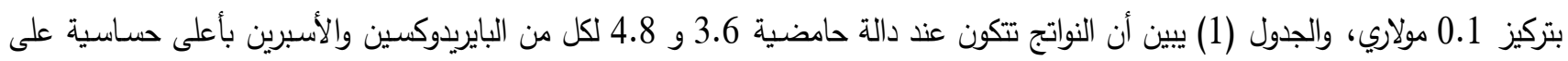
التوالي وبغياب الحامض، لذا تم استبعاد الحامض في طريقة العمل. لـان. جدول (1): تأثير كمية الحامض على امتصاص الأسبرين والبايريدوكسين هيلروكلوريد

\begin{tabular}{|c|c|c|c|c|}
\hline \multirow{2}{*}{$\begin{array}{c}\mathbf{H C l} \\
\mathbf{0 . 1} \mathbf{M}, \mathbf{m I}\end{array}$} & \multicolumn{2}{|c|}{ Absorbance } & \multicolumn{2}{c|}{$\mathbf{p H}$} \\
\cline { 2 - 5 } Without & Pyridoxine & Aspirin & Pyridoxine & Aspirin \\
\hline $\mathbf{0 . 5}$ & 0.250 & 0.321 & 3.6 & 4.8 \\
\hline $\mathbf{1 . 0}$ & -0.033 & -0.039 & 2.9 & 3.1 \\
\hline $\mathbf{1 . 5}$ & -0.049 & -0.072 & 2.5 & 2.9 \\
\hline $\mathbf{2}$ & -0.0523 & -0.095 & 2.4 & 2.5 \\
\hline $\mathbf{3}$ & -0.0345 & -0.045 & 2.0 & 2.4 \\
\hline $\mathbf{4}$ & -0.0246 & -0.010 & 1.9 & 2.3 \\
\hline $\mathbf{5}$ & 0.015 & -0.003 & 1.8 & 2.2 \\
\hline
\end{tabular}


لذا حضـرت محاليل منظمة مختلفة بقيمة 4.8 و3.8 للأدوية سـالفة الذكر على التوالي والجدول (2) يوضـح أن تلك المحاليل المنظمة أدت إلى تحطم الناتج أيضا حيث كان تأثيرها سلبياً على امتصاص كلا الدوائين لذا تم استبعادها في التجارب اللاحقة. جدول (2 ): تأثير المحاليل المنظمة على امتصاص الأسبرين والبايريدوكسين هيدركلوريد

\begin{tabular}{|c|c|c|}
\hline \multirow{2}{*}{ Buffer solution } & \multicolumn{2}{|c|}{ Absorbance } \\
\cline { 2 - 3 } & Pyridoxine (pH3.6) & Aspirin (pH4.8) \\
\hline Without & 0.252 & 0.320 \\
\hline Phthalate & 0.152 & 0.012 \\
\hline Glycine & 0.165 & 0.002 \\
\hline Acetate & 0.125 & 0.023 \\
\hline Citrate & 0.077 & 0.014 \\
\hline
\end{tabular}

تأثير تركيز الكاشف 9-كلورواكربين وحجمه

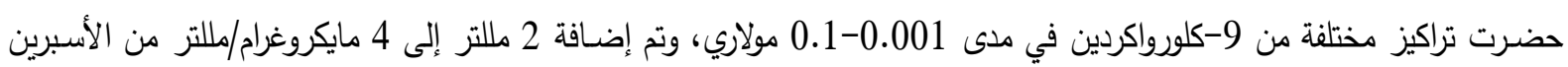

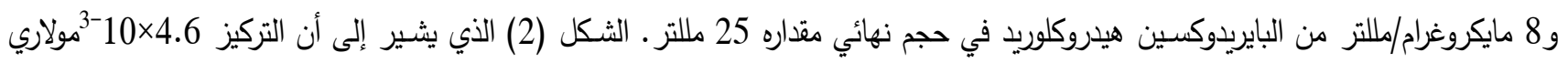
أعطى أعلى شدة امتصاص لكل من الأسبرين والبايريدوكسين هيدروكلوريد، لذا تم اعتماده في الاراسات اللاحقة.

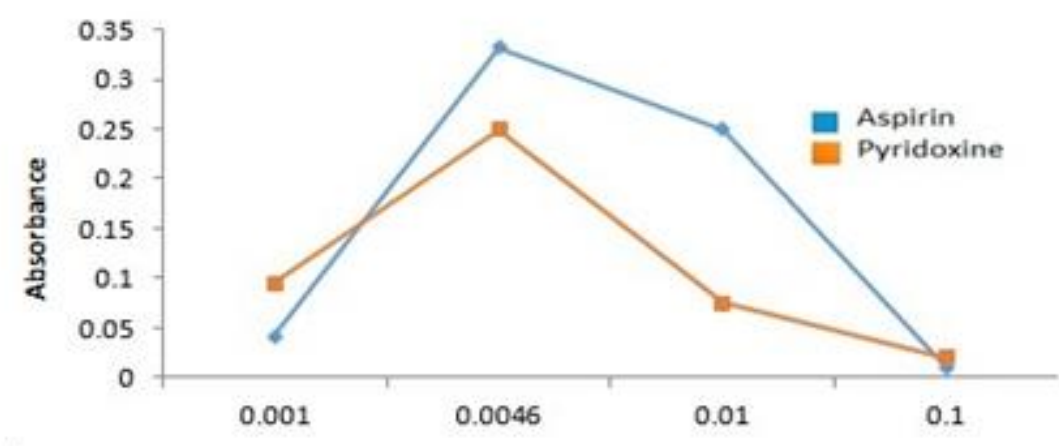

9-chloroacridin $\mathbf{M}, 2 \mathrm{ml}$

شــكل (2): تاثير تركيز الكاشــف 9-كلورواكريلين على امتصـــاص 4 مايكروغرام/مللتر اسـبرين و8 مايكروغرام/ ملتر بايريدوكسـين هيدروكلوريا.

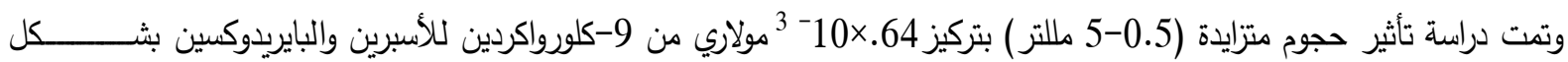

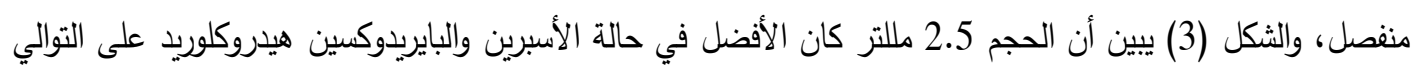




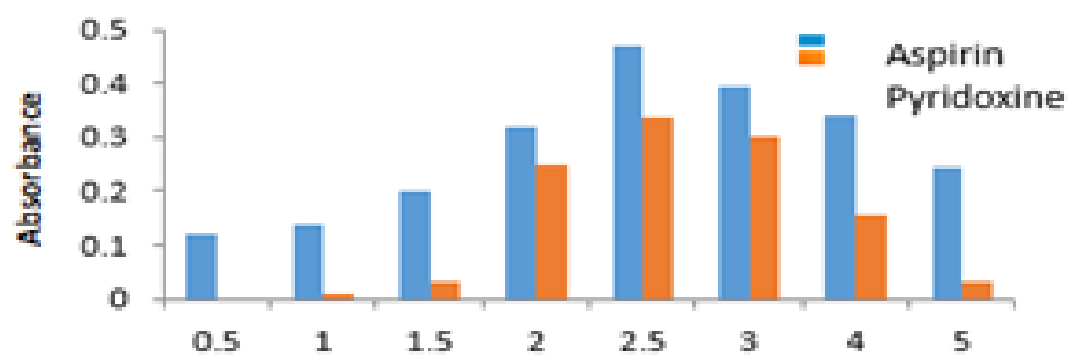

9-chloroacridin, $4.6 \times 10^{-3} \mathrm{M} \mathrm{ml}$

شكل (3): تاثير حجم الكاشف 9-كلورواكريدين على امتصاص 4 مايكروغرام/ ملتر اسبرين و8 مايكروغرام/ مللتر بايريدوكسين. تأثير المواد الفعالة سطعياً

لغرض زيادة الحساسية درس تأثير عدد من المواد الفعالة سطحياً المتضمنة توين-80 وصوديوم دوديكايل سلفيت(SDS) وترايتون X - 100وتوين -20 بحجم 1.0 مللتر وبتركيز 0.1\% لكل منها، وأدرجت النتائج في الجدول (3) الذي يوضح التأثير السلبي لتلك المواد على شدة الامتصاص جميعا وعليه تم استبعادها في الدراسات اللاحقة.

جلول (3) تأثير المواد الفعالة سطحيا

\begin{tabular}{|c|c|c|}
\hline \multirow{2}{*}{ Surfactant 0.1\% } & \multicolumn{2}{|c|}{ Absorbance } \\
\cline { 2 - 3 } & Aspirin & Pyridoxine \\
\hline Without & 0.470 & 0.335 \\
\hline Tween-80 & 0.200 & 0.202 \\
\hline Tween-20 & 0.244 & 0.125 \\
\hline SDS & 0.276 & 0.220 \\
\hline Triton X-100 & 0.108 & 0.105 \\
\hline
\end{tabular}

تأثير درجة الحرارة والزمن على امتصاص الناتج المتكون

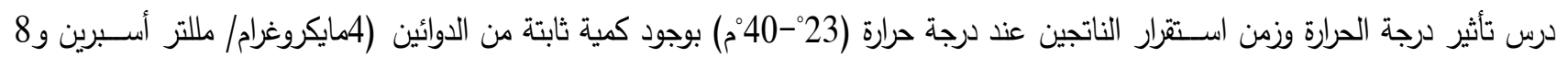

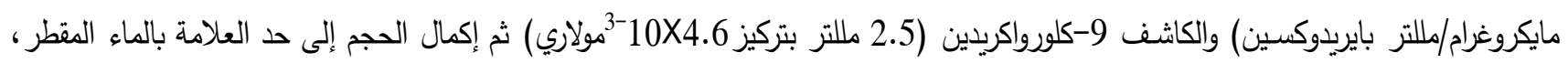

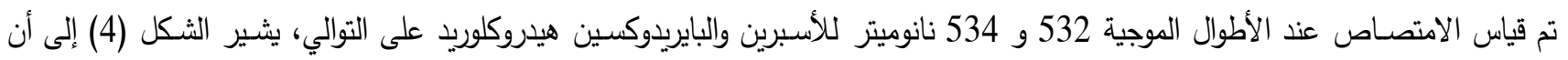

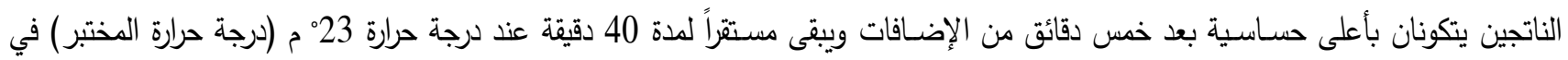
حالة الأسبرين في حين يبقى مستقراً لأكثر من 80 دقيقة عند نفس الارجة المذكورة في حالة البايريدوكسين. 

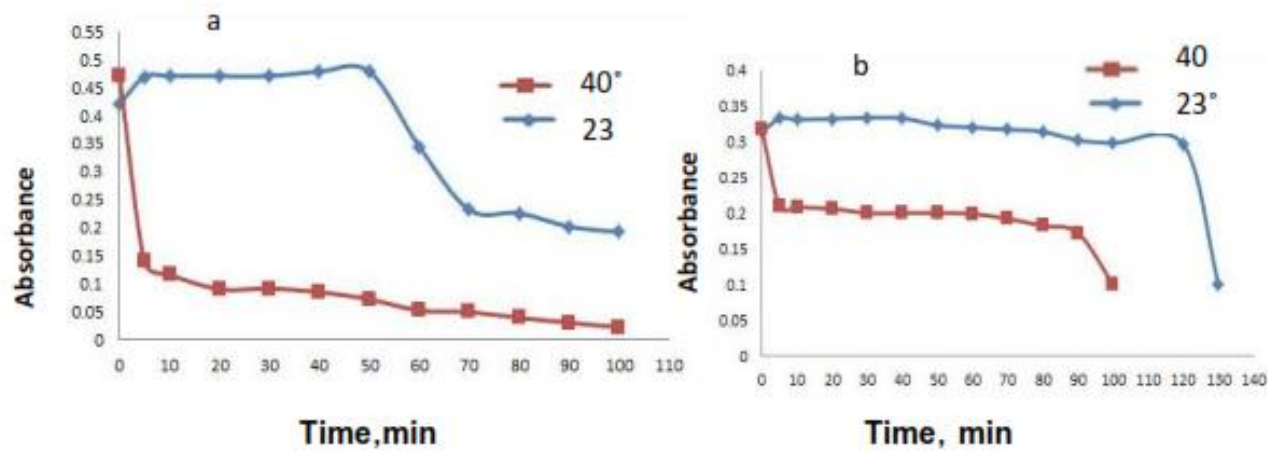

شكل (4): تأثير درجة الحرارة وزمن الاستقرار على امتصاص4 مايكروغرام/ مللتر اسبرين (a) 8 مايكرو غرام / ملتتر والبايريدوكسين (b) مع الكاثف 9-كلورو اكريلين.

$$
\text { مدى التقاير الخطي }
$$

تم الحصــول على منحنيات قياســية تتبع قانون بير ضـــمن المدى (0.2-18) و (0.4-24) مايكروغرام/مللتر لكل من الأسـبرين

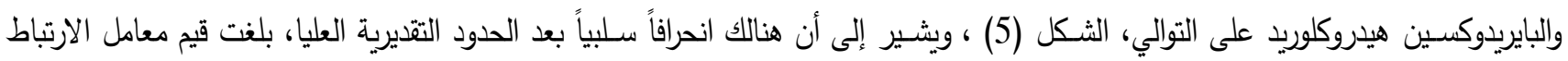

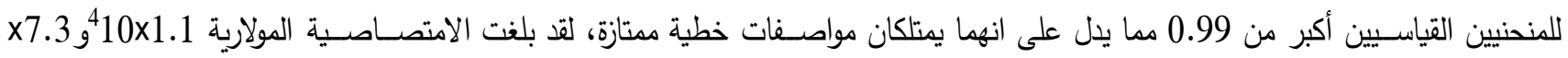

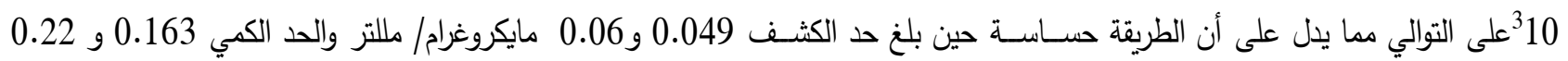

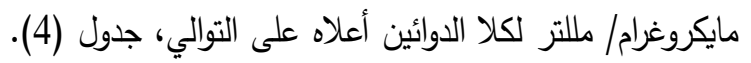
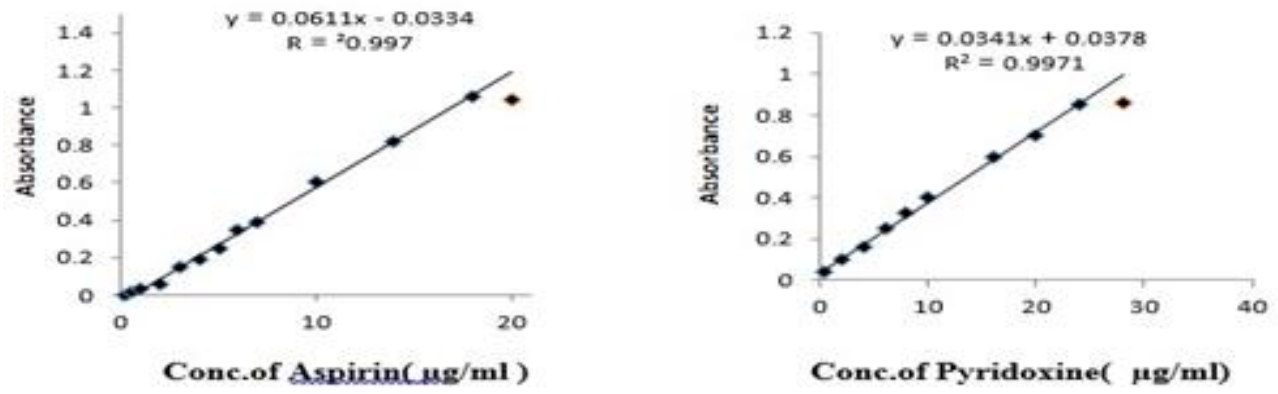

شكل (5): المنحنيات القياسية للاسبرين والبايريلوكسين 
جدول (4): الددى التقديري والإمتصاصية المولارية وحدود الكثف والددود الكمية لتقدير الأسبرين والبايريدوكسين هيدروكلوريد

\begin{tabular}{|l|c|c|}
\hline \multicolumn{1}{|c|}{ Parameters } & Aspirin & Pyridoxine \\
\hline Linearity range $\boldsymbol{\mu g} / \mathbf{m l}$ & $0.2-18$ & $0.4-24$ \\
\hline Molar absorptivity $\left(\mathbf{L} \cdot \mathbf{~ m o l}^{\mathbf{- 1}} \mathbf{c m}^{-1}\right)$ & $1.1 \times 10^{4}$ & $7 \times 10^{3}$ \\
\hline Intercept & 0.0334 & 0.0378 \\
\hline Slope & 0.0611 & 0.0341 \\
\hline Correlation coefficient $\left(\mathbf{R}^{2}\right)$ & 0.997 & 0.9971 \\
\hline LOD $(\boldsymbol{\mu g} / \mathbf{m l})$ & 0.049 & 0.06 \\
\hline LOQ $(\boldsymbol{\mu g} / \mathbf{m l})$ & 0.163 & 0.22 \\
\hline
\end{tabular}

دقة الطريقة وتوافقها

فحصت دقة الطريقة وتوافقها من خلال احتسـاب نسبة الاسـترجاع والانحراف القياسي النسبي باسـتخدام أربعة مكررات لثلاثة تراكيز

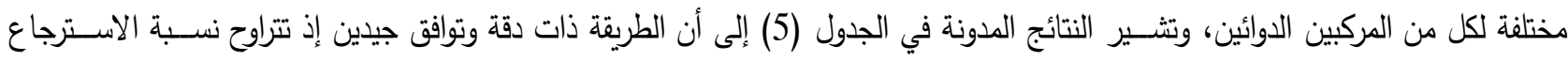

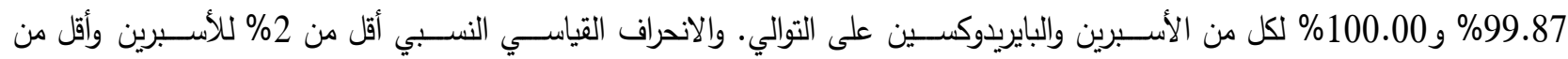
1.1 اللبايريدوكسين هيدروكلوريد مما يدل على توافقية الطريقة

جدول (5) دقة الطريقة وتوافقها

\begin{tabular}{|c|c|c|c|c|}
\hline Drug & $\begin{array}{l}\text { Amount } \\
\text { added } \\
(\mu \mathrm{g} / \mathrm{ml})\end{array}$ & $\begin{array}{c}\text { Recovery } \\
\text { (\%) }\end{array}$ & $\begin{array}{c}\text { Average } \\
\text { recovery (\%) }\end{array}$ & RSD* \\
\hline \multirow[t]{3}{*}{ Aspirin } & 1 & 100 & \multirow{3}{*}{99.87} & 1.9 \\
\hline & 7 & 99.74 & & 0.33 \\
\hline & 14 & 99.87 & & 0.121 \\
\hline \multirow{3}{*}{ Pyridoxine } & 2 & 99 & \multirow{3}{*}{100.00} & 1.01 \\
\hline & 8 & 100.6 & & 0.94 \\
\hline & 20 & 100.42 & & 0.81 \\
\hline
\end{tabular}

${ }^{\star}$ Average of four determinations.

تأثير المتداخلات

تم دراسـة تأثير عدد من مواد السـواغ التي تذخل في المسـتحضــرات الصـيدلانية للتأكد من أن الطريقة انتقائية وخالية من التداخلات وذلك

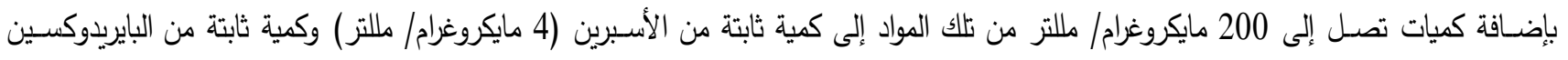

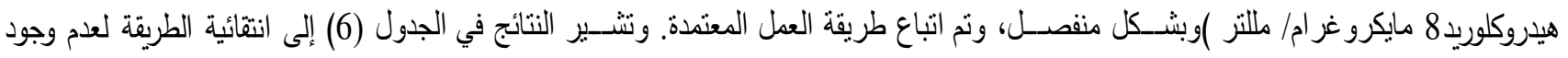


جلول (6) تأثير المتداخلات

\begin{tabular}{|c|c|c|c|c|c|c|}
\hline \multirow{3}{*}{ Excipient } & \multicolumn{6}{|c|}{ Recovery \% } \\
\hline & \multicolumn{3}{|c|}{$\begin{array}{c}4 \mu \mathrm{g} / \mathrm{ml} \text { of Aspirin } \\
\text { Per } \mu \mathrm{g} / \mathrm{ml} \text { of excipient }\end{array}$} & \multicolumn{3}{|c|}{$\begin{array}{c}\text { 8} \mu \mathrm{g} / \mathrm{ml} \text { of Pyridoxine hydrochloride } \\
\text { Per } \mu \mathrm{g} / \mathrm{ml} \text { of excipient }\end{array}$} \\
\hline & 50 & 100 & 200 & 50 & 100 & 200 \\
\hline Glucose & 99.99 & 100 & 94.70 & 99.69 & 101.53 & 104.61 \\
\hline Lactose & 100 & 100 & 92.06 & 99.69 & 104.30 & 98.15 \\
\hline Acacia & 100 & 99.47 & 98.94 & 99.38 & 101.53 & 61.53 \\
\hline Starch & 100 & 100 & 100 & 104.30 & 99.69 & 98.15 \\
\hline $\mathrm{NaCl}$ & 100 & 100 & 92.59 & 99.38 & 101.53 & 99.38 \\
\hline
\end{tabular}

\section{تقييم نتائج الطريقة المطورة}

تم تطبيق الطريقة بنجاح لتقدير الأسبرين على هيئة حبوب والبايريدوكسين على هيئة حبوب وحقن، جدول (7). ومن أجل إثبات نجاح

الطريقة المطورة وكفاءتها في تقدير الأسـبرين والبايريدوكسين هيدروكلوريد في مستحضـراتهما الصـيدلانية بشكل أقراص، فقد أجريت مقارنة بين الطريقة المطورة (المقترحة) وطريقة الدستور البريطاني (30) ، إذ اعتدت الطريقة في تقدير الأسبرين على تسحيح الفائض من هيدروكسيد الصوديوم مع حامض الهيدروكلوريك بوجود دليل الفينولفثالين، في حين اعتمدت الطريقة في تقدير البايريدوكسـين (31) على إضـــافة حامض الهيدروكلوريك وقياس الامتصـاص عند 290 نانوميتر • وذلك باسـتخدام اختباري t و بتطبيق القوانين الإحصـائية ، ويمكن الإسـتنتاج من النتائج المدونة في الجدول (8) على أن قيمةt التجريبية هي أقل من قيمة t الجدولية (2.571) عند مستوى ثقة 95 \% ولستة درجات حرية مما يدل على أن الطريقة المقترحة موثوق بها وصالحة في التطبيق على المستحضرات الصيدلانية، كما وجد أن قيمة التجريبية هي أقل من قيمتها الجدولية (9.28) عند مســتوى ثقة 95\% ولثلاث درجات حرية, وهذا يدل على أنه لا يوجد فرق معنوي بين الطريقتين وأن الطريقة المقترحة موثوق بها وصـــالحة في التطبيق على المستحضرات الصيدلانية. 
Journal of Education and Science (ISSN 1812-125X), Vol: 29, No: 1, 2020 (60-74)

$$
\text { الجدول (7) تقدير المركبات الدوائية في المستحضرات الصيدلانية }
$$

\begin{tabular}{|c|c|c|c|c|c|c|}
\hline $\begin{array}{c}\text { Pharmaceutical } \\
\text { Preparation }\end{array}$ & $\begin{array}{l}\text { Certified } \\
\text { value } \\
\text { (mg) }\end{array}$ & $\begin{array}{l}\text { Amount } \\
\text { present } \\
(\mu \mathrm{g} / \mathrm{ml})\end{array}$ & $\begin{array}{c}\begin{array}{c}\text { Amount } \\
\text { found }\end{array} \\
(\mu \mathrm{g} / \mathrm{ml})\end{array}$ & $\begin{array}{c}\text { Recovery } \\
(\%)\end{array}$ & $\begin{array}{c}\text { Average } \\
\text { recovery } \\
(\%)\end{array}$ & $\begin{array}{c}\text { Average } \\
\text { recovery } \\
(\mathrm{mg})\end{array}$ \\
\hline \multirow{3}{*}{ Aspirin $^{a}$} & \multirow{3}{*}{75} & 1 & 1.03 & 103 & \multirow{3}{*}{100.37} & \multirow{3}{*}{75.27} \\
\hline & & 7 & 6.89 & 98.42 & & \\
\hline & & 14 & 13.96 & 99.71 & & \\
\hline \multirow{3}{*}{ Aspirin ${ }^{b}$} & \multirow{3}{*}{100} & 1 & 1.05 & 105 & \multirow{3}{*}{100.66} & \multirow{3}{*}{100.66} \\
\hline & & 7 & 6.81 & 97.27 & & \\
\hline & & 14 & 13.96 & 99.71 & & \\
\hline \multirow{3}{*}{ Aspirin $^{c}$} & \multirow{3}{*}{81} & 1 & 1.05 & 105 & \multirow{3}{*}{100.73} & \multirow{3}{*}{81.59} \\
\hline & & 7 & 6.94 & 99.14 & & \\
\hline & & 14 & 13.73 & 98.07 & & \\
\hline \multirow{3}{*}{ Injection $\left(B_{6}\right)^{c}$} & \multirow{3}{*}{100} & 2 & 1.97 & 98.5 & \multirow{3}{*}{99.35} & \multirow{3}{*}{99.35} \\
\hline & & 8 & 8.2 & 102.5 & & \\
\hline & & 20 & 19.41 & 97.05 & & \\
\hline \multirow{3}{*}{ Pyridoxine $^{a}$} & \multirow{3}{*}{50} & 2 & 2.1 & 105 & \multirow{3}{*}{101.56} & \multirow{3}{*}{50.78} \\
\hline & & 8 & 8.2 & 102.5 & & \\
\hline & & 20 & 19.4 & 97.2 & & \\
\hline
\end{tabular}

${ }^{\text {a }}$ UK, ${ }^{\mathrm{b}}$ SDI, ${ }^{\mathrm{c}}$ India

جدول (8) تقييم نتائج الطريقة المطورة للأسبرين والبايريدوكسين هيدركلوريد

\begin{tabular}{|c|c|c|c|c|c|}
\hline \multirow{2}{*}{ Drug } & \multirow{2}{*}{$\begin{array}{c}\text { Pharmaceutical } \\
\text { preparation }\end{array}$} & \multicolumn{2}{|c|}{ Recovery (\%) } & \multirow{2}{*}{$t_{\text {exp }}$} & \multirow{2}{*}{$F_{\text {test }}$} \\
\cline { 3 - 5 } & Aspesent & $\begin{array}{c}\text { Standard } \\
\text { method }\end{array}$ & & \\
\hline Aspirin & Aspirin tablet & 100.66 & 101.34 & 1.299 & 8.49 \\
\hline Pyridoxine & SamavitB & 99.65 & 98.86 & 2.04 & 2.435 \\
\hline
\end{tabular}


دراسة طبيعة المقثّ وثثابت استقراريته

تم اتباع طريتتي التغيرات المستمرة ونسبة الميل (29,28) للناتج المتكون بين الأسبرين والبايريدوكسين هيدروكلوريد مع 9-كلورواكريدين

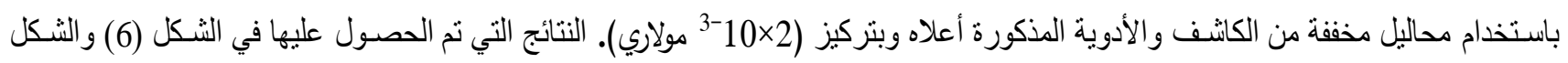

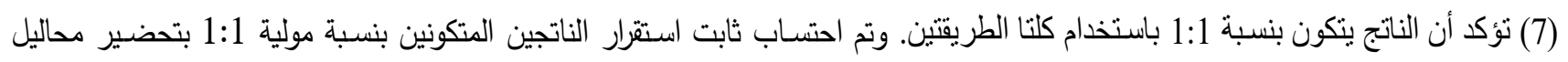

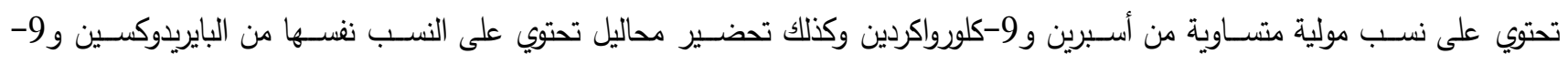

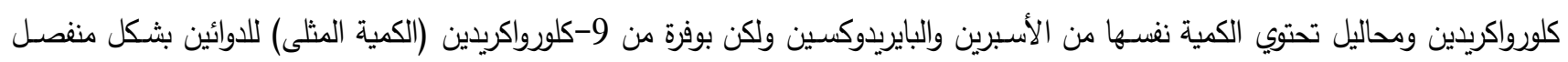
بتطبيق العلاقة الأتية:

$$
\begin{aligned}
& \propto=A m-A s / A m \\
& k_{s t}=\frac{1-\propto}{\alpha^{2} c}
\end{aligned}
$$

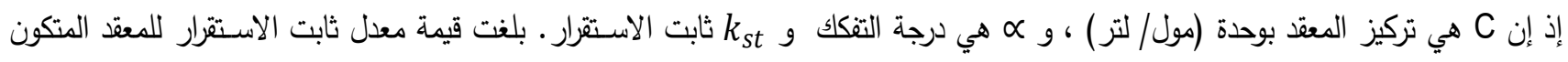

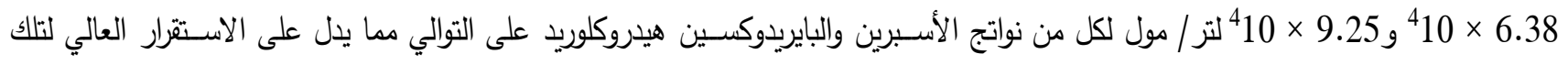

النواتج جدول (9) (9)
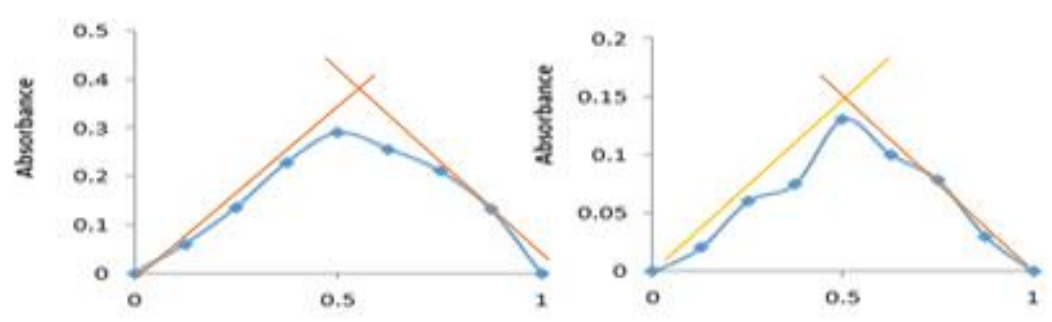

[Aspirin] $/[9-\mathrm{CA}]+[9-\mathrm{CA}] \quad$ [Pyridoxine] $/[9-\mathrm{CA}]+[9-\mathrm{CA}]$

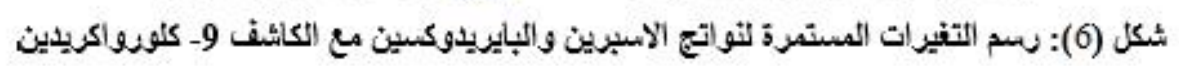
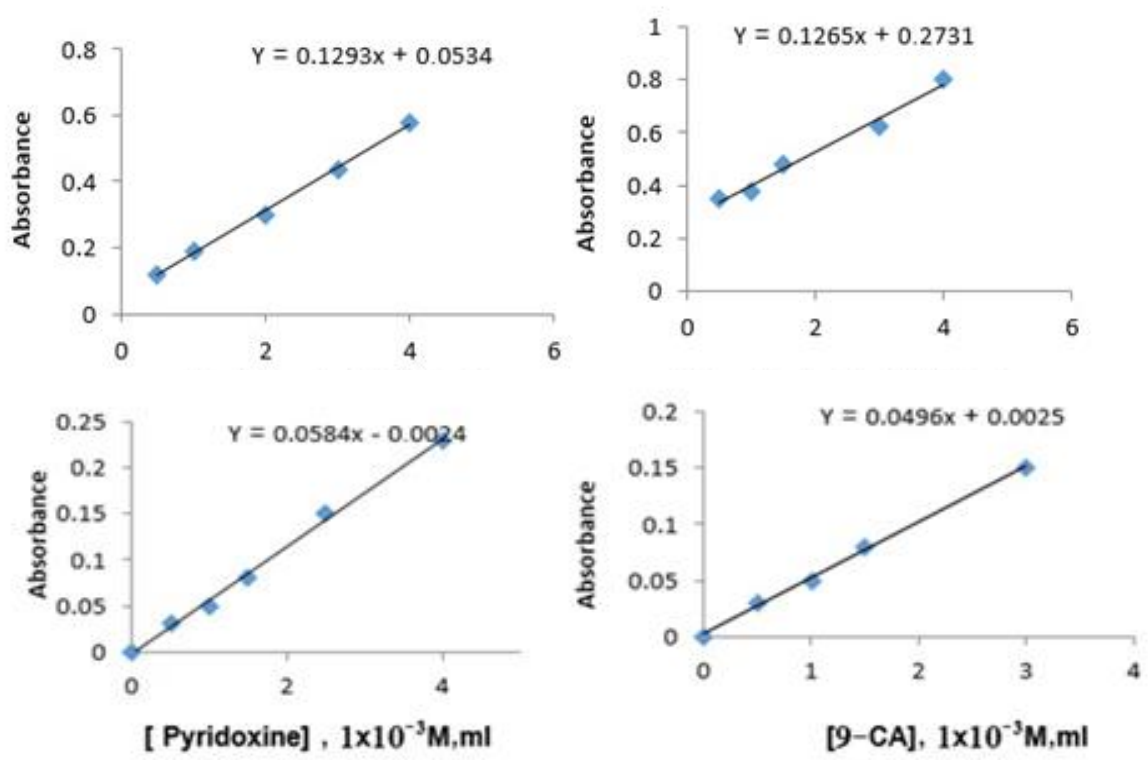

شكل (7): رسم نسبة الميل لنواتج الأسبرين والبايريدوكسين مع الكاشف 9ـ كلورواكريدين 
التفاعل الكيميائي المقترح

من خلال التركيب الكيميائي للمركب الدوائي الأسبرين يتوقع حدوث تحلل مائي للأسبرين في الوسط المائي لينتج حامض السـالسليك

وحامض الخليك، إذ يتم حدوث مهاجمة نيوكلوفيلية على الكاربون رقم9 في الكاشــف 9-كلورواكريدين وعند طرح جزيئة حامض الهيدروكلوريك

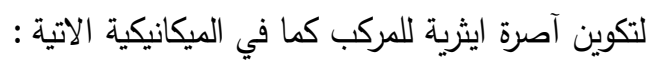<smiles>CC(=O)Oc1ccccc1C(=O)O[Na]</smiles><smiles>O=C(O)c1ccccc1Oc1c2ccccc2nc2ccccc12</smiles>

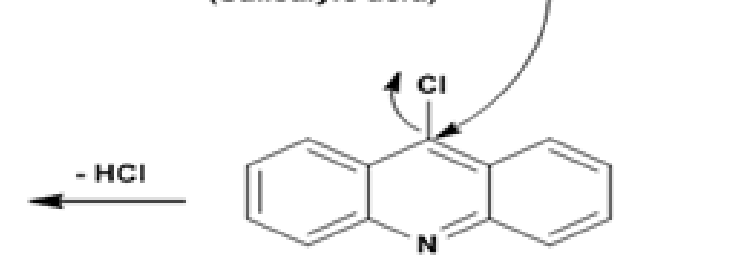<smiles>CC(=O)CCc1ccccc1C(=O)O</smiles>

(Salisalyic acid)

9-chloroacridine

أما بالنسـبة للمركب الدوائي الثاني وهو البايريدوكسـين فنلاحظ وجود مجموعة النتروجين في الحلقة الاروماتية التي بدورها تعمل على تحفيز مجموعة

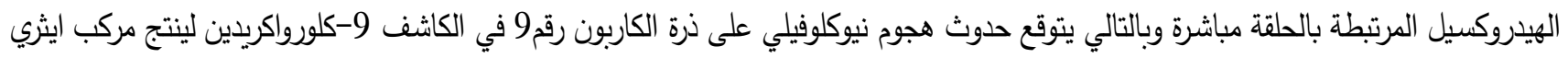

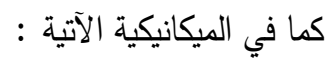<smiles>Cc1ncc(CO)c(CO)c1O</smiles>

Pyridoxint 9-chloroacridine<smiles>Cc1ncc(CO)c(CO)c1Oc1c2ccccc2nc2ccccc12</smiles>

[5-(acridin-9-yloxy|-6-methylpyridine-3,4diylydimethanol 
جلول (9) ثابت استقرار مققدي الأسبرين والبايريدوكسين هيدوكلوريد

\begin{tabular}{|c|c|c|c|c|c|}
\hline \multirow{2}{*}{ Compound } & \multirow{2}{*}{ Conc. (mol/l) } & \multicolumn{2}{|c|}{ Absorbance } & \multirow{2}{*}{$\alpha$} & \multirow{2}{*}{$\begin{array}{c}\text { Average } \mathrm{K}_{\mathrm{s}} \\
(\mathrm{I} / \mathrm{mol})\end{array}$} \\
\hline & & As & $A m$ & & \\
\hline \multirow{3}{*}{ Aspirin } & $8.0 \times 10^{-5}$ & 0.102 & 0.372 & 0.725 & \multirow{3}{*}{$6.38 \times 10^{4}$} \\
\hline & $1.2 \times 10^{-4}$ & 0.325 & 0.508 & 0.360 & \\
\hline & $1.6 \times 10^{-4}$ & 0.503 & 0.620 & 0.188 & \\
\hline \multirow{3}{*}{ Pyridoxine } & $4.0 \times 10^{-5}$ & 0.040 & 0.060 & 0.333 & \multirow{3}{*}{$9.25 \times 10^{4}$} \\
\hline & $8.0 \times 10^{-5}$ & 0.080 & 0.120 & 0.333 & \\
\hline & $1.2 \times 10^{-4}$ & 0.100 & 0.150 & 0.333 & \\
\hline
\end{tabular}

الاستنتاج

تم تطوير طريقة طيفية بسيطة لتقدير الأسبرين والبايريدوكسين هيدروكلوريد. اعتمدت الطريقة على تفاعل التعويض النيكوفيلي بين الدوائين والكاثـف 9 -كلورواكريدين وقياس الناتج المتكون عند 532 و534 نانوميتر لكلا الدوائين على التوالي، لقد أمكن تتدير بحدود 0.2-18 و 0.424 مايكروغرام/مللتر من الأسبرين والبايريدوكسين وبدقة جيدة إذ بلغ معدل نسبة الاسترجاع 99.87\%و 100.00\% وتوافق أقل من2\% للأسبرين وأقل من 1.2\% للبايريدوكسين هيدروكلوريد على التوالي، طبقت الطريقة بنجاح في تقدير الدوائيين في مستحضريهما الصيدلانية. كما وجد من خلال دراسة طبيعة المعقد أن المعقد يتكون بنسبة 1:1 دواء:9-كلورواكريدين، كما تم مقارنة الطريقة المطورة مع طريقة الدستور البريطاني للأدوية، تميزت الطريقة المطورة بالحساسية وبالسهولة وعدم الحاجة إلى استخلاص أو تسخين وفي المحلول المائي فضلاًعن كونها في درجة حرارة الغرفة.

\section{المصادر}

1. Goodman, L. S., Gilman, A., Brunton, L. L., Hilal-Dand an, R., \& Knollmann, B. C. 11th ed 404-1119. (2018).

2. Stationery Office. British pharmacopoeia 2009. London, Stationery Office. (2008).

3. Malti Sharma, Mallika Pathak, Bani Roy, Lovely Jain, Nikanshi Yadav, Bidushi Sarkar, Milanpreet Kaur, and Mansi Sharma. DU Journal of Undergraduate Research and Innovation 157-162 (2015).

4. Veeresh T. M. ,Shekappa Lamani ,Sharanappa T Nandibewoor. Transition Metal Chemistry34(3):317-324 (2009).

5. Bnar M. Ibrahim. Journal of Raparin University.3. 6. (2016).

6. Nikanshiyadavd, Bidushi Sarkar, Milanpreet Kaur and Mansi. DU Journal of Undergraduate Research and Innovation, 157-162. (2015).

7. Maruf, A. Journal of Medical Sciences (Faisalabad). 1, 61-62(2001), 
8. Mitić, S. S., Miletić, G. Z., Pavlović, A. N., Tošić, S. B., \& Sunarić, S. Acta Chimica Slovenica. 55, 508-515. (2008).

9. F Lirato, A. Al-Bassam, N Al-Sabea. Iraqi Journal of Market Research and Consumer Protection 2 (4): 182-195. (2010).

10. Ramjith,U., sunith, D., Radhakrishnan, S., \& Sameer, P. Brazilian Journal of Pharmaceutical Sciences. ,45, 4. (2013).

11. De Aguiar J.L.N., Leandro K.C., Abrantes S.D.M.P., \& Albert A.L.M. Revista Brasileira De Ciencias Farmaceuticas/Brazilian Journal of Pharmaceutical Sciences. 45, 723-727. (2009).

12. Mcmahon, G. P., Connor, S. J., Fitzgerald, D. J., Le Roy, S., \& Kelly, M. T. Journal of Chromatography B. 707, 322-327. (1998).

13. Saeed, A. M., Hamzah, M. J. And Ahmed, N. Q. International Journal of Applied Pharmaceutics, 10(5), 167172. (2018).

14. Jabbar, H. Science Journal of University of Zakho. 4, 94-103. (2016).

15. Mokhtari, Ali, Keyvanfard, Mohsen, Emami, Iraj, Delouei, Nastaran J., Pishkhani, Hatameh F., Ebrahimi, Aida, \& Karimian, Hossein. Journal of the Brazilian Chemical Society, 27(3), 566-574. (2016).

16. Raza, A., Ansari, T. M., Niazi, S. B., \& Rehman, A. Journal of the Chemical Society of Pakistan. 29, 33-36. (2007).

17. Srividya K, \& Balasubramanian N. Journal of JAOAC International. 80,6,1368-1373. (1997).

18. N. A. Obed-Agha (2010), Ph.D. Thesis, College of Education for Pure Sciences., University of Mosul

19. Asma N. Abdul Kadir Rafidain Journal of Science. 21 ,4, 49-59. (2010)

20. J. G. Portela, A. C. S. Costa, and L. S. G. Teixeira. Journal of Pharmaceutical and Biomedical Analysis, 34, no. 3, 543-549. (2004).

21. Rocha, F. R., Fatibello Filho, O., \& Reis, B. F. Talanta. 59, 191-200. (2002).

22. Consigliere, V. O., Vals, N. R. M., \& Magalhaes, J. F. Pharmaceutical Analytical Letters. 34, 1875-1888. (2001).

23. Kuzmanović, Darko; Khan, Muslim; Mehmeti, Eda; Nazir, Ruqia; Amaizah, Naser Ramdan R.; Stanković, Dalibor M.Diamond and Related Materials, 64, 184-189. (2016).

24. Cottica, Solange M., Nozaki, Jorge, Nakatani, Helena S., Oliveira, Claudio C., Souza, Nilson E. De, \& Visentainer, Jesui V. Journal of the Brazilian Chemical Society, 20(3), 496-501. (2009).

25. S.Ravichand ran, S.Selvakumar, Afreen, Nuzhat Banu .Indian Journal of Pharmaceutical Science \& Research , $8,1,35-43 .(2018)$.

26. Kompantseva, E. V., Khalata, A. V., Ovcharenko, L. P., Dukkardt, L. N., \& Blagorazumnaya, N. V. Pharmaceutical Chemistry Journal .39, 8, 441-443. (2005).

27. Anyakora, C., Afolami, I., Ehianeta, T.S., \& Onwumere, F. African Journal of Pharmacy and Pharmacology 2(2):29-36. (2008). 
28. Willard, H. H. Instrumental Methods of Analysis. New Delhi, CBS.

29. Harvey, A. E., \& Manning, D. L. Journal of the American Chemical Society. 72, 4488-4493. (1950).

30. British Pharmacopoeia Commission, \& Great Britain. British pharmacopoeia 2013. London, Stationery Office. (2012).

31. Al- Shreify Z.A.M.M.Sc. Thesis, College of Education for Pure Sciences., University of Mosul (2012). 